International Journal of Biomedicine I June 2019 - Volume 9, Issue Suppl_1:

Abstracts From the Second Russian International Conference "Cryo-electron microscopy 2019: achievements and prospects"

\author{
ORAL ABSTRACT PRESENTATIONS \\ SESSION TITLE: MOLECULAR ORGANIZATION OF CELLS AND ORGANELLES
}

DOI: 10.21103/IJBM.9.Suppl_1.OR8

\title{
Abstract OR-8: High-Resolution Structures of Microtubule-Drug Complexes Revealed by Cryo-EM
}

$\underline{\text { Kushal Sejwal }}^{1}$, Thorsten B. Blum ${ }^{1,2}$, Joseph Atherton ${ }^{3}$, Ashwani Sharma ${ }^{1}$, Natacha Olieric ${ }^{1}$, Andrea E. Prota $^{3}$, Carolyn A. Moores ${ }^{3}$, Michel O. Steinmetz ${ }^{1,2}$

${ }^{1}$ Laboratory of Biomolecular Research, Division of Biology and chemistry, Paul Scherrer Institute, Villigen, Switzerland; ${ }^{2}$ University of Basel, Biozentrum, Basel, Switzerland; ${ }^{3}$ Institute of Structural and Molecular Biology, Birkbeck, University of London, London, United Kingdom

Background: Microtubules are dynamic protein filaments that are crucial for cell division and constitute key elements of the cytoskeleton. They are assembled from $\alpha \beta$-tubulin heterodimers that form hollow cylindrical structures. There is a large number of naturally occurring compounds that are known to interact with tubulin, including alkaloids, macrolides and peptides, which are collectively called microtubule-targeting agents (MTAs). Based on their activities, MTAs can be classified as microtubulestabilizing agents (MSAs) that enhance MT assembly, and microtubule-destabilizing agents (MDAs) that suppress MT assembly. The chemical structure of these drugs and their binding mode to microtubules varies greatly amongst each other and confer the ability to act either synergistically or competitively on microtubules. Owing to their effects on microtubule dynamics, MTAs are of great interest and widely used in a variety of medical applications as antiparasitic agents, herbicides and, most importantly, as chemotherapeutic drugs used for the treatment of cancer.

In the past years, we and others solved the structures of a large number of different MTAs bound to tubulin to high resolution using X-ray crystallography. Very recently, however, with the advent of the "Resolution Revolution" in cryo-electron microscopy (cryo-EM), atomic structures of known MSAs bound to microtubules have also been obtained. These cryo-EM structures confirmed that the sites and modes of binding described in the previous X-ray crystallographic studies are similar in the context of the assembled microtubule but additionally explain the effects of MTAs on lattice parameters in microtubules and the lateral contacts between protofilaments, especially at the microtubule seam.

Methods: Cryo-EM and model building.

Results: We solved several high-resolution structures of microtubules bound to novel MSAs and identified differential binding modes in comparison to that revealed by X-ray crystallography.

Conclusion: Cryo-EM and X-ray crystallography can be used in a complementary manner to investigate the molecular mechanism of action of MSAs in detail. 
Key Words: cryo-EM • microtubule $\bullet$ microtubule-stabilizing agents

International Journal of Biomedicine. 2019;9 Suppl 1: S9. doi: 10.21103/IJBM.9.Suppl_1.OR8

(C)2019 International Medical Research and Development Corporation 